\title{
Supplementary material to \\ Not ions alone: Barriers to ion permeation in nanopores and channels
}

\author{
Oliver Beckstein, Kaihsu Tai, and Mark S. P. Sansom \\ Department of Biochemistry, University of Oxford, South Parks Road, Oxford OX1 3QU, UK
}

August 5, 2004

\section{1 lons in wide pores}

It seems surprising that ions are considerably affected even in a pore of $2 \mathrm{~nm}$ diameter, greater than its length of $1.6 \mathrm{~nm}$, and about five times the ionic diameter. The radially averaged density from the MD simulations shows that water already behaves rather bulk like in the pore [Fig. S1(a)]. It fills almost the whole pore volume at bulk density. Near the pore surface the same characteristic water layers are visible that are also seen at the slab-reservoir interface. The ionic density, Fig. S1(b), is concentrated in the central region and only reaches about $0.9 \mathrm{~mol} \cdot \mathrm{L}^{-1}$, i.e. $70 \%$ of the bulk value. This effect is not due to insufficient sampling time as investigation of the density plots from shorter stretches of the $100 \mathrm{~ns}$ trajectory indicates. (About $20 \mathrm{~ns}$ appear to be the minimum time necessary to obtain meaningful densities).

The effect of the surface on the ions extends noticeably for at least $1 \mathrm{~nm}$. This is also borne out by the radial distribution function $g\left(r ; \mathrm{Na}^{+}, \mathrm{O}_{\mathrm{w}}\right)$ (Fig. S2) from a homogeneous electrolyte $\left(99 \mathrm{~ns} \mathrm{MD}\right.$ at $1 \mathrm{~mol} \cdot \mathrm{L}^{-1} \mathrm{NaCl}$ concentration in a $4 \times 4 \times 4 \mathrm{~nm}^{3}$ simulation box, $T=300 \mathrm{~K}$, $P=1$ bar). The first water peak of height 7.59 is at a distance of $0.226 \mathrm{~nm}$ from the $\mathrm{Na}^{+}$center but the second peak is still notable (1.44) at $0.447 \mathrm{~nm}$. The first hydration shell of $\mathrm{Na}^{+}$contains about 4.6 water molecules in a radius of $0.32 \mathrm{~nm}$, while the second shell adds another 15.6 within $0.53 \mathrm{~nm}$ (by reading off the number of water molecules $N(r)$ within a sphere of radius $r$ at the minimum following a peak in $g(r)$ (see the inset of Fig. S2). Thus about 20 water molecules in a sphere of radius $0.53 \mathrm{~nm}$ are noticeably influenced by a $\mathrm{Na}^{+}$ion. On the other hand, water layering effects near the hydrophobic surface extend over two to three layers of water, ${ }^{1}$ influencing water $0.6 \mathrm{~nm}$ afar from the surface. Taken together, this suggests a range of the wall-ion interaction of about $1 \mathrm{~nm}$, mediated through the structure of the solvent-a effect which is clearly not captured by the PB calculations, and which would be also absent in Brownian dynamics simulations of the system.

\section{Wall flexibility}

The local flexibility of the wall of the model pores can be adjusted by changing the harmonic spring constant $k$ which restrains each pore pseudo atom to its initial position (see Section 3.1). We previously discussed that increased wall flexibility, i.e. a softer wall, decreased the permeability for water molecules. ${ }^{1}$ We carried out PMF calculations for water in the $R=0.55 \mathrm{~nm}$ pore for differing $k$. The permeation barrier follows the very same trend as the water permeability. ${ }^{1}$ Fig. S3 shows results for a softer and a more rigid pore than the ones used in the main paper. The local flexibility of the wall atoms is measured by their positional root mean square deviation (RMSD). For the standard pore with $k=k_{0}=1.0 \times 10^{3} \mathrm{~kJ} \cdot \mathrm{mol}^{-1} \cdot \mathrm{nm}^{-2}=4 \times 10^{2} k T \mathrm{~nm}^{-2}$, the RMSD is $0.072 \mathrm{~nm}$. By increasing the local flexibility to an RMSD of $0.17 \mathrm{~nm}\left(k=0.2 k_{0}\right)$ the barrier to permeation increases substantially from $\Delta G^{\ddagger}\left(k=k_{0}\right)=3.2 k T$ by $7.2 k T$ to $\Delta G^{\ddagger}\left(k=0.2 k_{0}\right)=10.5 k T$. However, making the pore wall twice as rigid (i.e. more crystalline, with RMSD $0.052 \mathrm{~nm}$ ) has a negligible effect; the barrier only drops by $0.4 k T$ to $\Delta G^{\ddagger}\left(k=2 k_{0}\right)=2.8 k T$. Even though at present we lack a theory that relates the PMF (or at least the barrier height) and the pore radius, length, and flexibility the simulations do show that the PMF reflects the profound influence that local flexibility can have on permeation properties of water molecules. Because ion permeation in hydrophobic pores requires the ion's water environment to be stable in the pore (see the main paper), we anticipate an even stronger effect on the ion PMF for softer pores. Calculations are planned to address this question in more detail. 

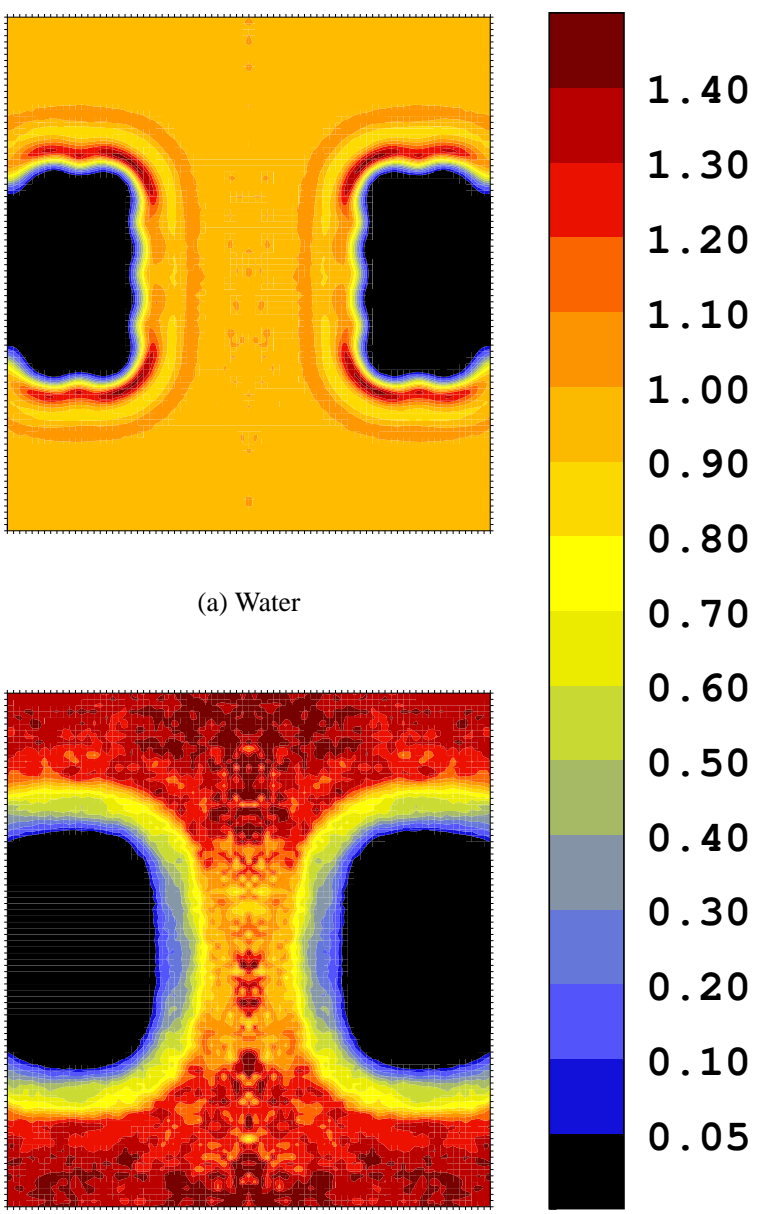

(a) Water

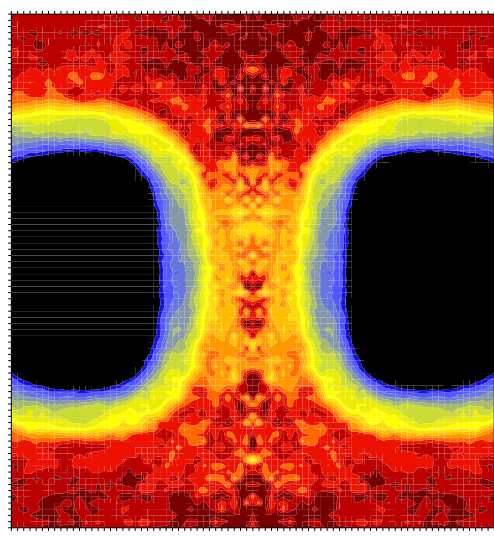

(b) $\mathrm{Na}^{+}$

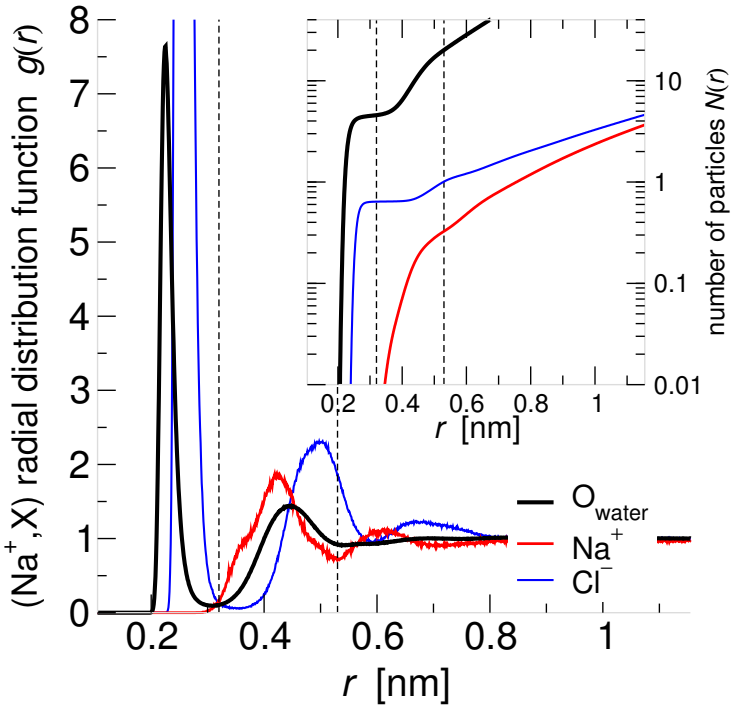

Figure S2: Radial distribution function $g(r)$, centered on $\mathrm{Na}^{+}$in $1 \mathrm{M} \mathrm{NaCl}$ bulk solution. Broken vertical lines indicate the boundary of the first and second hydration shell of a sodium ion, taken as the minima in $g\left(r ; \mathrm{Na}^{+}, \mathrm{O}_{\mathrm{w}}\right)$. The inset shows the number of particles of a given type inside a sphere of radius $r$ around a $\mathrm{Na}^{+}$ ion, $N(r)=n_{0} \int_{0}^{r} d r^{\prime} 4 \pi r^{\prime 2} g\left(r^{\prime}\right)$.

one layer of pseudo-atoms, and radius $1.0 \mathrm{~nm}$ ) at either end of the constriction site of length $L=0.8 \mathrm{~nm}$ and radius $R$ (varied between $0.15 \mathrm{~nm}$ and $1.0 \mathrm{~nm}$ ). The pore is embedded in a membrane mimetic, a slab of pseudo-atoms held on a cubic lattice with unit cell length $0.39 \mathrm{~nm}$ with harmonic springs of strength $k_{0}$.

\subsection{Molecular dynamics} pore with radius $R=1.0 \mathrm{~nm}$. The color scale indicates the local concentration in mol. $\mathrm{L}^{-1}$ for $\mathrm{Na}^{+}$or units of the bulk density of SPC water $n_{0}=1.0 \mathrm{~g} \cdot \mathrm{cm}^{-3}$ at $T=300 \mathrm{~K}$ and $P=1 \mathrm{bar}$. (The figures were mirrored on the pore axis to create the impression of a full pore; they were created with XFARBE ${ }^{3}$ )

\section{Methods}

\subsection{Pore model}

The pore models were constructed as described previously. ${ }^{2}$ Briefly, they consist of concentric rings of methane-like pseudo-atoms of van der Waals radius $0.195 \mathrm{~nm}$. These are held in their equilibrium position by harmonic restraints with spring constant $k_{0}=1000 \mathrm{~kJ} \cdot \mathrm{mol}^{-1} \cdot \mathrm{nm}^{-2}$, resulting in average positional RMSDs of $0.072 \mathrm{~nm}$, which is similar to the root mean square fluctuations of backbone atoms of about $0.1 \mathrm{~nm}$ observed in MD simulations of ion channels. ${ }^{4}$ A pore consists of two mouth regions (length $0.4 \mathrm{~nm}$, i.e.
MD simulations were performed with GROMACS version 3.2.1 ${ }^{5}$ and the SPC water model. ${ }^{6}$ The Lennard-Jones parameters for the interaction between a methane-like pseudoatom and the water oxygen are $\varepsilon_{\mathrm{CO}}=0.906493 \mathrm{~kJ} \cdot \mathrm{mol}^{-1}$ and $\sigma_{\mathrm{CO}}=0.342692 \mathrm{~nm}$; parameters for sodium and chloride ions are taken from the GROMACS force field. The integration time step was 2 fs and coordinates were saved every 2 ps. Simulations were carried out for at least $100 \mathrm{~ns}$. With periodic boundary conditions, long range electrostatic interactions were computed with a particle mesh Ewald method (real space cutoff $1 \mathrm{~nm}$, grid spacing $0.15 \mathrm{~nm}$, 4th order interpolation $^{7}$ ) while the short range van der Waals forces were calculated within a radius of $1 \mathrm{~nm}$. The neighbor list (radius $1 \mathrm{~nm}$ ) was updated every 10 steps. Weak coupling algorithms ${ }^{8}$ were used to simulate at constant temperature $(T=300 \mathrm{~K}$, time constant $0.1 \mathrm{ps})$ and pressure $\left(P=1\right.$ bar, compressibility $4.5 \times 10^{-5}$ bar $^{-1}$, time constant $1 \mathrm{ps)} \mathrm{with} \mathrm{the} x$ and $y$ dimensions of the simulation cell held fixed at $3.9 \mathrm{~nm}$. The total thickness of the wa- 


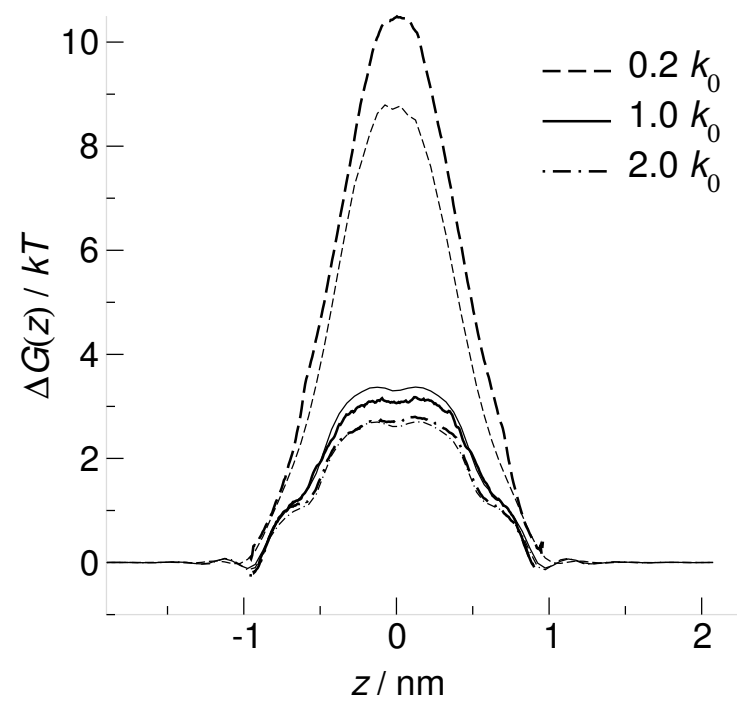

Figure S3: PMF of a water molecule in pores of varying local flexibility at the fixed nominal radius $R=0.55 \mathrm{~nm}$. The flexibility of the pore wall atoms is set through the harmonic force constant $k$ of the restraint force. Calculations in the main paper are reported for $k=k_{0}$, with $k_{0}=1.0 \times 10^{3} \mathrm{~kJ} \cdot \mathrm{mol}^{-1} \cdot \mathrm{nm}^{-2}$. The umbrellasampled PMF is given by the thick line; the PMF derived from the equilibrium density is shown as a thin line of the same line style.

ter reservoir in the $z$-direction was $3.0 \mathrm{~nm}$, ensuring bulklike water behaviour far from the membrane mimetic. The initial system configuration was created by solvating the slab-embedded pore model with water. For simulations with ions, some water molecules were replaced with ions to reach the target concentration. Initially there were always ions present in pores with $R \geq 0.4 \mathrm{~nm}$. A simulation box measured $3.9 \times 3.9 \times 4.6 \mathrm{~nm}^{3}$ and contained about 1500 water molecules and 280 pseudo-atoms (and between 25 to $28 \mathrm{Na}^{+}$and $\mathrm{Cl}^{-}$ions each).

\subsection{Potential of mean force}

The PMF is related to the equilibrium density $n(\mathbf{r})$ through the density of the reference state in the bulk far from the pore $n_{0}$,

$$
\beta G(\mathbf{r})=-\ln \frac{n(\mathbf{r})}{n_{0}}+\beta G_{0}
$$

(where $\beta^{-1}=k T$ ). The free energy of the reference state $G_{0}$ is an unknown constant. If sampling is sufficient during the course of a MD trajectory one can directly calculate $\Delta G(z)=G(z)-G_{0}$ from the equilibrium density $n(\mathbf{r})$ (see Fig. S1) using Eq. (1).

Alternatively, one can bias the equilibrium distribution of a test ion to the energetically unfavorable regions using the technique of umbrella sampling. ${ }^{9}$ We restrained a $\mathrm{Na}^{+}$ion or water molecule with a harmonic potential
$\left(U(z)=k / 2\left(z-z_{i}\right)^{2}, k=7693 \mathrm{~kJ} \cdot \mathrm{mol}^{-1} \cdot \mathrm{nm}^{-2}\right)$ at subsequent equally spaced positions $z_{i}$ along the $z$ axis of the pore [spacing $\left.\left(z_{i}-z_{i-1}=0.025 \mathrm{~nm}\right)\right]$. This restricted its movement to the $x y$-plane while still allowing diffusion into adjacent windows. For each window a starting frame from an equilibrium trajectory was selected which had the particle in question (ion or water molecule) close to $z_{i}$ (maximum initial deviation was $0.3 \mathrm{~nm}, 80 \%$ of initial distances were smaller than $0.1 \mathrm{~nm}$ ). One MD simulation of length $0.6 \mathrm{~ns}$ was carried out for each of the 101 windows, using the same parameters as above. The positional deviation $\xi(t)=z(t)-z_{i}$ of the particle was recorded for every step (2 fs). A correlation time $\int d t\langle\xi(t) \xi(0)\rangle /\left\langle\xi^{2}\right\rangle$ of typically $0.2 \mathrm{ps}$ indicates that we actually oversampled $\xi$.

The 101 biased distributions of $z$ positions of the test particle (ion or water) were recombined and unbiased with the Weighted Histogram Analysis Method (WHAM) ${ }^{10,11}$ The first $0.1 \mathrm{~ns}$ of each window run were discarded as equilibration time, leaving a total of $0.5 \mathrm{~ns}$ per window. A tolerance of at least $10^{-5} k T$ was required in the self-consistent WHAM procedure.

The PMF in the bulk and mouth region of the pore is obtained from the equilibrium MD trajectories. Umbrella sampled PMFs are only meaningful in regions where the diffusion of the particle is bounded in the $x y$-plain, i.e. the pore and mouth region. Final PMF profiles were assembled by matching equilibrium profiles and umbrella sampled profiles in the mouth region where both methods are accurate.

For ions in pores with $R \geq 0.6 \mathrm{~nm}$ only equilibrium MD is necessary to collect the complete PMF; sufficient sampling is achieved in $100 \mathrm{~ns}$. Water PMFs could be obtained from equilibrium MD reliably for all radii except for the $R=0.15 \mathrm{~nm}$ pore but for comparison the PMF for a water molecule in the $R=0.55 \mathrm{~nm}$ pore was calculated. The umbrella sampled water PMFs were symmetrized as they exhibited a small linear skew of about $1 k T$. The symmetrized water PMF in the $R=0.55 \mathrm{~nm}$ pore is almost identical to the one computed from the equilibrium density (Figure S4). Although similar "hysteresis" effects have been reported ${ }^{12}$ and attributed to correlations in subsequent starting configurations, their origin is not clear in our case as the starting positions in adjacent windows tend to be from different times of the equilibrium trajectory and are therefore uncorrelated. The WHAM procedure itself was tested with simulated input based on a flat underlying PMF and a step barrier of height $2 k T$. Both "PMFs" could be recovered completely within $0.05 k T$ of the exact result.

\subsection{Poisson-Boltzmann calculations}

Poisson-Boltzmann calculations were carried out with APBS. ${ }^{13}$ The test $\mathrm{Na}^{+}$ion was assigned a Born radius of 


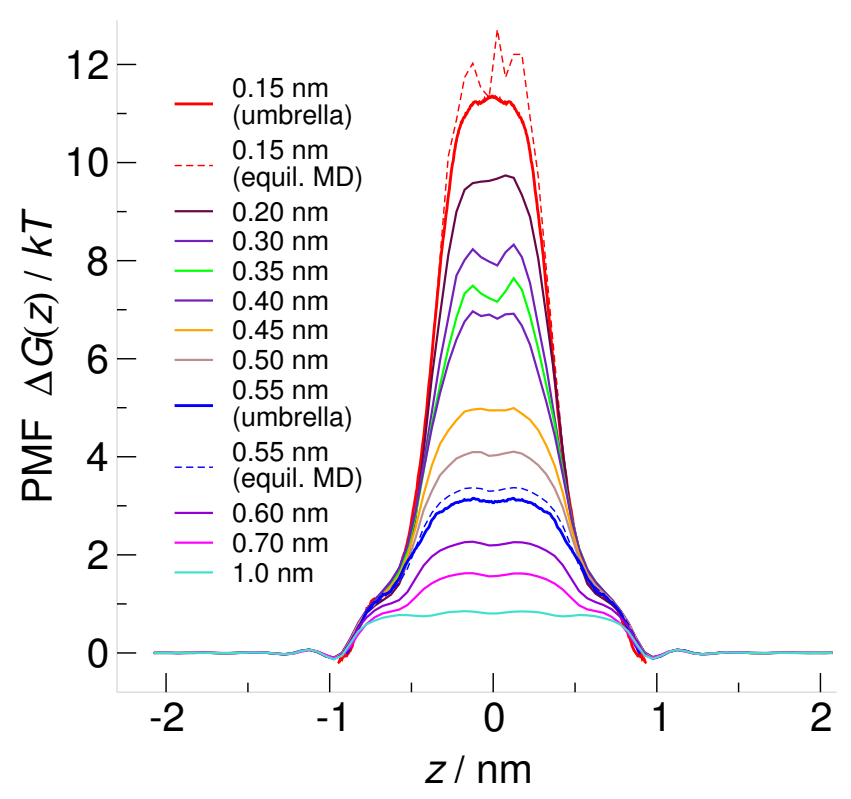

Figure S4: PMF profile for water. The PMF was derived from equilibrium simulations except for the $R=0.15 \mathrm{~nm}$ and $0.55 \mathrm{~nm}$ pore, where umbrella sampling was employed. In both cases, the equilibrium-density derived PMF is also shown for comparison.

$0.1680 \mathrm{~nm} .{ }^{14}$ The electrostatic binding energy of the ion was calculated at subsequent positions $z$ as

$$
\Delta G_{B}(z)=G_{\text {pore }+ \text { ion }}(z)-G_{\text {ion }}-G_{\text {pore }}
$$

The radius of a (implicit) solvent molecule was set to $0.14 \mathrm{~nm}$, the approximate radius of a water molecule.

Further parameters were chosen as to be consistent with the MD simulations. The temperature was $300 \mathrm{~K}$, the ionic strength $1.3 \mathrm{M} \mathrm{NaCl}$. The water dielectric constant $\varepsilon_{\mathrm{w}}$ was 63 (the value of the SPC water model ${ }^{15}$ ), instead of the experimental value $\varepsilon_{\mathrm{w}}=78.5$. The dielectric constant $\varepsilon_{\mathrm{p}}$ of the pore and slab material was set to 1 because in the MD simulations the material is modeled as simple van der Waals spheres without partial charges which can not screen any electric field and hence are best described by $\varepsilon_{\mathrm{p}}=1$; a more realistic value for hydrocarbons would be $\varepsilon_{\mathrm{p}}=2$.

In addition to the calculations at a concentration of $1.3 \mathrm{~mol} \cdot \mathrm{L}^{-1} \mathrm{NaCl}$ we also performed the same calculations at $0 \mathrm{~mol} \cdot \mathrm{L}^{-1} \mathrm{NaCl}$ and $7 \mathrm{~mol} \cdot \mathrm{L}^{-1} \mathrm{NaCl}$. The binding energy $\Delta G_{B}$ shows negligible sensitivity on to the ionic strength. This is sensible because the test ion is the only charge in the system. Two sets of calculations with water dielectric coefficient at different values (63 for the SPC water model and 78.5 as the experimental value at $300 \mathrm{~K}$ ) also indicated negligible sensitivity thereto. The calculations were sensitive to the grid discretization necessary to solve the Poisson-Boltzmann equation by a finite element scheme. Our tests on grid sizes ranging from 65 to 129 grid points indicated that our results are converged for grids of size $97 \times 97 \times 97$, using a focusing scheme (final resolution $0.031 \mathrm{~nm})$ and a smoothed dielectric boundary.

Acknowledgments. We thank Nathan Baker for help with APBS and Born energy calculations, Graham Smith and Marc Baaden for useful discussions about umbrella sampling and WHAM.

\section{References}

1. Beckstein, O.; Sansom, M. S. P. Physical Biology 2004, 1, 42-52.

2. Beckstein, O.; Sansom, M. S. P. Proc. Natl. Acad. Sci. USA 2003, 100, 7063-7068.

3. Preusser, A. ACM Trans. Math. Softw. 1989, 15, 79-89.

4. Domene, C.; Grottesi, A.; Sansom, M. S. P. Biophys. J. 2004, 87, 256-267.

5. Lindahl, E.; Hess, B.; van der Spoel, D. J. Mol. Mod. 2001, 7, 306-317.

6. Hermans, J.; Berendsen, H. J. C.; van Gunsteren, W. F.; Postma, J. P. M. Biopolymers 1984, 23, 1513-1518.

7. Darden, T.; York, D.; Pedersen, L. J. Chem. Phys. 1993, 98, 10089-10092.

8. Berendsen, H. J. C.; Postma, J. P. M.; DiNola, A.; Haak, J. R. J. Chem. Phys. 1984, 81, 3684-3690.

9. Valleau, J. P.; Torrie, G. M. A Guide to Monte Carlo for Statistical Mechanics: 2. Byways. In Statistical Mechanics. Part A: Equilibrium Techniques, Vol. 5; Berne, B. J., Ed.; Plenum Press: New York, 1977.

10. Kumar, S.; Bouzida, D.; Swendsen, R. H.; Kollman, P. A.; Rosenberg, J. M. J. Comp. Chem. 1992, 13, 1011-1021.

11. Roux, B. Comp. Phys. Comm. 1995, 91, 275-282.

12. Roux, B.; Karplus, M. Biophys. J. 1991, 59, 961-981.

13. Baker, N. A.; Sept, D.; Joseph, S.; Holst, M. J.; McCammon, J. A. Proc. Natl. Acad. Sci. USA 2001, 98, 10037-10041.

14. Rashin, A. A.; Honig, B. J. Chem. Phys. 1985, 89, 5588-5593.

15. van der Spoel, D.; van Maaren, P. J.; Berendsen, H. J. C. J. Chem. Phys. 1998, 108, 10220-10230. 